\title{
The Top 100 Most-Cited Papers in Erosive Tooth Wear: A Bibliometric Analysis
}

\author{
Aurélio de Oliveira Rocha ${ }^{a}$ Pablo Silveira Santos ${ }^{a}$ Bárbara Azevedo Machado ${ }^{a}$ \\ Michele Bolan ${ }^{\mathrm{a}}$ Mariane Cardoso ${ }^{\mathrm{a}}$ Paulo Antônio Martins-Júnior ${ }^{\mathrm{b}}$ \\ Carla Miranda Santana ${ }^{a}$ \\ ${ }^{a}$ Department of Dentistry, Federal University of Santa Catarina (UFSC), Florianópolis, Brazil; ${ }^{b}$ Department of \\ Paediatric Dentistry, Federal University of Minas Gerais (UFMG), Belo Horizonte, Brazil
}

\section{Keywords}

Bibliometrics - Databases · Bibliography · Publications ·

Tooth erosion

\begin{abstract}
Citation rates can be used as an indicator of the influence and relevance of scientific papers. The present study analyzed the 100 most-cited articles related to erosive tooth wear (ETW). The top 100 most-cited papers focusing on ETW topics were collected from the Web of Science database on November 11, 2020. The following bibliometric data were extracted from papers: title, authorship, institutions, countries, number of citations, year of publication, journal title, study design, topic, and keywords. Scopus and Google Scholar were searched to compare the number of citations. The VOSviewer software was used to generate collaborative network maps for the authors and keywords. The number of citations of the 100 most-cited papers ranged from 71 to 330 (average: 97.44). The papers were published between 1949 and 2015. Caries Research (28\%) and The Journal of Dentistry (16\%) presented the majority of papers. Laboratorial (44\%) and observational studies (33\%) were the most common study designs. The most studied topics were epidemiology
\end{abstract}

(31\%) and the erosive potential of substances (drinks, foods, or medicines) (22\%). The countries with the highest number of most-cited papers were England (32\%), Germany (18\%), and Switzerland (10\%). The University of Bern was the institution with the most papers (10\%). Lussi A was the author with the highest number of papers in the top 100 (14\%). The most common keyword was "dental erosion" with 64 occurrences. The top 100 most-cited papers related to ETW were composed mainly of laboratorial and observational studies focusing on epidemiology and the erosive potential of substances.

(c) 2022 S. Karger AG, Basel

\section{Introduction}

Dental erosion consists of the chemical loss of mineralized tooth substances caused by exposure to nonbacterial intrinsic or extrinsic acids [Schlueter et al., 2020]. Erosive tooth wear (ETW) refers to tooth wear with dental erosion as the primary etiological factor [Schlueter et al., 2020]. The prevalence of ETW was 30.4\% in permanent teeth of children and adolescents [Salas et al., 2015]. Its diagnosis comprises the assessment of patients' his- 
tory, predisposing factors, and a clinical examination. Typical early signs of ETW include shallow and shiny defects, usually on smooth surfaces. However, flattening and cupping can be observed on occlusal surfaces [Schlueter et al., 2020]. In severe cases, ETW can result in aesthetic and functional impairment. Dentin hypersensitivity can be a significant issue, directly reducing patients' oral health-related quality of life [Schlueter et al., 2012].

The term bibliometrics was first proposed by Alan Pritchard in the late 1960s and was defined as applying statistical and mathematics methods to books. Bibliometric studies map existing concepts, usually through citations, pointing out the primary theoretical lenses used to investigate a subject and raising available methodological tools [Kear, and Colbert-Lewis, 2011]. Citation rates are widely used as an indicator of the relevance and merit of papers and scientific journals. A high number of citations may indicate a paper's potential to influence clinical decisions and future research [Hutchins et al., 2016; Perazzo et al., 2019; Ahmad et al., 2020]. Bibliometric analysis can help researchers identify research trends, study designs, topics covered, prominent authors, leading scientific journals, and countries with the highest publication performance [Jayaratne, and Zwahlen, 2015].

Several dentistry fields and topics have been addressed in bibliometric studies to date [Perazzo et al., 2019; Patil et al., 2020; Baldiotti et al., 2021]. However, to the best of our knowledge, no bibliometric analysis of papers related to ETW has yet to be conducted. Such a study may assist researchers to identify topics and research trends related to ETW, identifying the main evidence and demonstrating knowledge gaps in the area, thus stimulating new investigations. Therefore, this bibliometric study aimed to analyze the characteristics of the top 100 most-cited papers related to ETW.

\section{Materials and Methods}

A list of papers focused on topics related to ETW was obtained from the WoS Core Collection (WoS-CC) database, based on a keyword search strategy (TS = ["tooth erosion" OR "dental erosion" OR "erosive tooth wear" OR "erosive tooth lesions" OR "dentine erosion" OR "enamel erosion" OR "dental corrosion”]) on November 11, 2020. Papers were displayed in descending order based on the number of citations. Two independent researchers (AOR and PSS) identified the 100 most-cited papers after reading the titles, abstracts, and full texts, when necessary. No limitations to the year of publication or language were applied. Conference papers and studies in which ETW was not the central topic were excluded. Any disagreement was resolved through discussion and consensus with a third researcher (CMS). A crossmatch with the number of citations for each paper was performed at Scopus and Google Scholar databases. In the event of a tie, the paper's position in the list was based on the highest WoS-CC citation density (average number of citations received per year), followed by the highest number of citations at Scopus.

Data extracted from papers were title, authorship, number of citations, citation density (number of citations per year), institution, countries and continent (based on the corresponding author's affiliation), year of publication, journal title, study design, topic, and keywords. Data were double-checked to avoid errors. Study designs were classified as reviews (systematic or nonsystematic), laboratorial (in vitro), controlled clinical simulations (in situ), observational, and interventional studies. All papers were grouped according to the most general topics: epidemiology, diagnosis, the erosive potential of substances (beverages, foodstuffs, or medications), saliva role on ETW, and anti-erosive/remineralizing agents. Topics that appeared only once were classified as "others."

The VOSviewer software was used to generate collaborative density maps between authors (only connected authors are presented) and between keywords (only keywords with at least three occurrences were included using the All Keywords option). Each point on the density map has a color that indicates the items' density at that point. Colors range from blue to yellow to red. The greater the number of items in the vicinity of a point and the greater the weight of neighboring items, the closer the color is to red. In contrast, the smaller the number of items in the vicinity of a point and the lower the weight of the neighboring items, the closer the color of the matter is to blue [Jan van Eck, and Waltman, 2017].

Data analysis was performed with the statistical software package SPSS for Windows (SPSS, version 24.0; IBM Corp). Kolmogorov-Smirnov test was used to verify the normality of data distribution. Spearman's rank correlation coefficient test was used since the data were not normally distributed.

\section{Results}

Initially, 1,936 documents were retrieved from WoSCC. After organizing the list in descending order of citations, 142 papers were screened for eligibility and 42 were excluded, resulting in the top 100 most-cited papers (online suppl. Table 1; for all online suppl. material, see www. karger.com/doi/10.1159/000521877). Reasons for exclusions were conference papers and studies that did not focus on topics related to ETW. Altogether, the top 100 papers were cited 9,744 times in the WoS-CC, including 522 self-citations (5.4\%). The number of citations ranged from 71 to 330 , and 39 papers were cited at least 100 times. The number of citations was higher in Scopus $(10,964$ citations) and Google Scholar (21,530 citations). There was a strong positive correlation between the number of citations in WoS-CC and Google Scholar $($ rho $=0.789)$ and a very strong correlation in WoS-CC and Scopus (rho = 0.945). Although significant, weak negative correlations were observed between the number of citations and the 


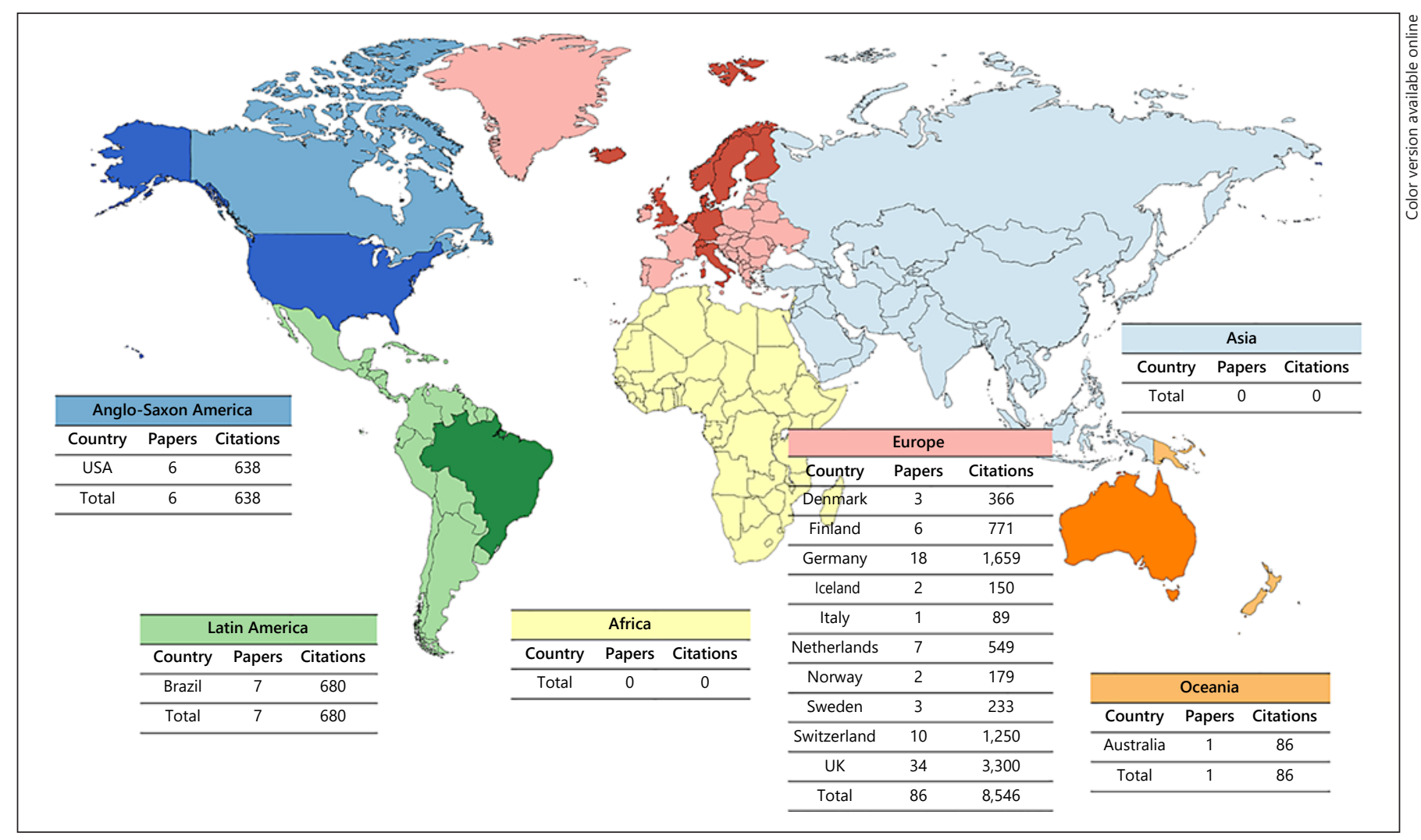

Fig. 1. Worldwide distribution of the top 100 most-cited papers related to ETW.

year of publication of the paper, in WoS-CC (rho = -0.220 ), Google Scholar (rho $=-0.248$ ), and Scopus $($ rho $=-0.282)$.

The most-cited paper was "Risk factors in dental erosion," written by Järvinen VK, Rytömaa II, and Heinonen OP, published in 1991, in The Journal of Dental Research [Jarvinen et al., 1991]. It was cited 330 times, with an average of 11.38 citations/year at WoS-CC. It was also the most-cited paper at Scopus (390 citations) and Google Scholar (746 citations). The oldest paper was published in 1949 [Zipkin, and Mcclure, 1949] and the most recent in 2015 [Carvalho et al., 2015]. Most studies were published in the 2000s (54\%), followed by the 1990s (27\%). The 100 most-cited papers were published mainly in Caries Research (28\%), followed by The Journal of Dentistry (16\%) (online suppl. Table 2). Laboratorial studies were the most common (44\%), followed by observational studies (33\%), controlled clinical simulations (10\%), and nonsystematic reviews $(8 \%)$. Interventional studies and systematic reviews constituted only $3 \%$ and $2 \%$ of the papers, respectively. Epidemiology (31\%), erosive potential of substances (drinks, foods, or medications) (22\%), anti-

The Top 100 Most-Cited Papers in Erosive Tooth Wear erosive/remineralizing agents (20\%), saliva's role in ETW (13\%), and diagnosis (5\%) were the main topics.

Europe hosted 86 of the 100 most-cited papers (Fig. 1) and, undoubtedly, had the highest number of citations $(8,546)$. The papers from Anglo-Saxon America were all from the USA ( 6 papers; 638 citations). Brazil represented Latin America (7 papers; 680 citations), and Australia represented Oceania with only one paper (86 citations). Asia and Africa had no papers in the top 100 list. At a country level, England had the highest number of papers and citations (32 papers and 3,106 citations). Germany (18 papers; 1,659 citations) and Switzerland (10 papers; 1,250 citations) also presented an expressive number of papers and citations. A total of 42 institutions participated in the 100 most-cited papers. The Universities of Bern (Switzerland), Bristol (England), and Birmingham (England) were the leading academic centers, with 10,9, and 7 papers, respectively (online suppl. Table 3 ).

Two hundred and thirty-three authors were identified in the 100 most-cited papers. A VOSviewer density map was generated to detail the collaborative coauthorship between the authors through the clusters (online suppl. Fig. 


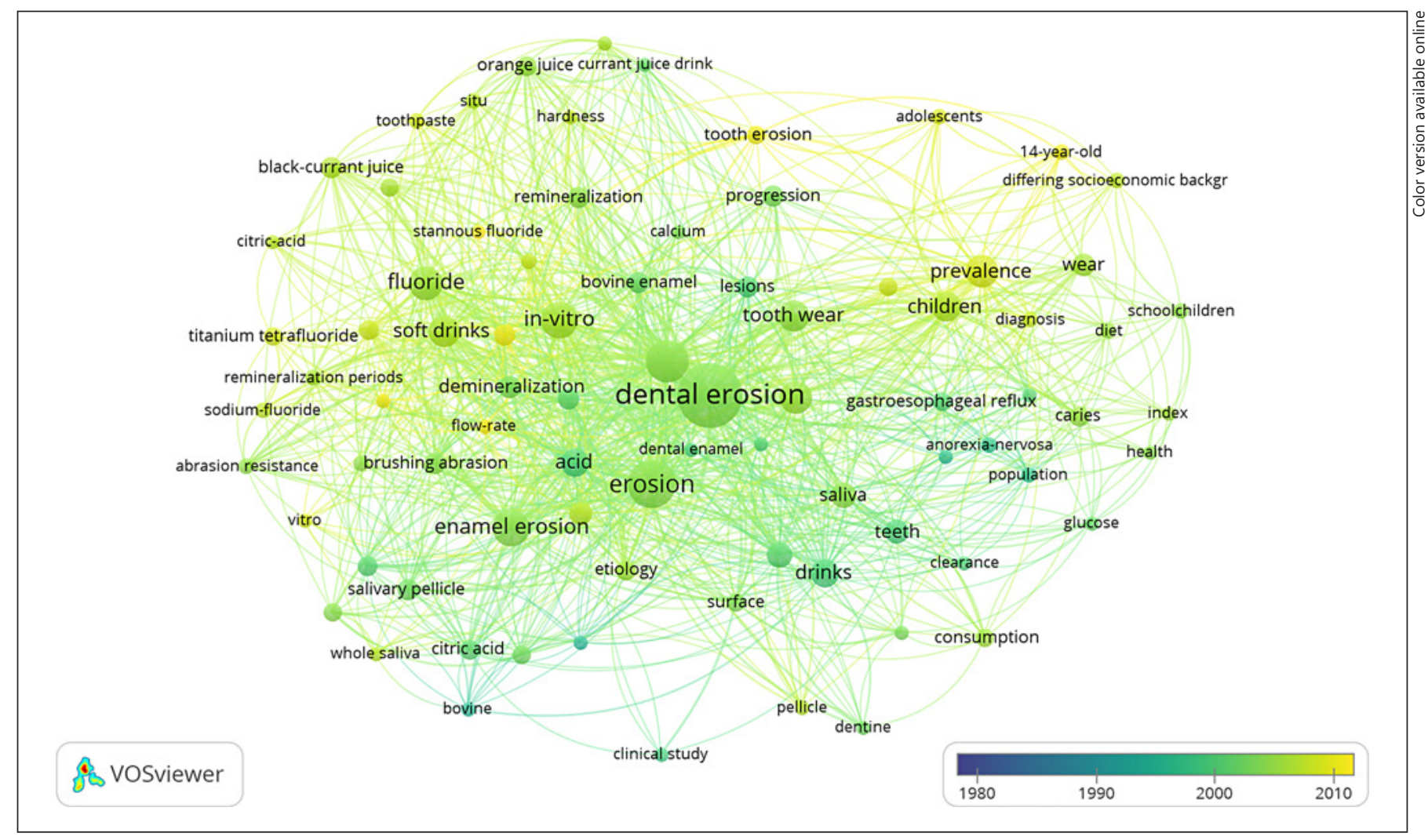

Fig. 2. Bibliographic clustering between keywords in the top 100 most-cited papers related to ETW (only keywords with at least 3 occurrences were included).

1). In total, 32 clusters of authors were identified and 10 presented 10 or more authors. The biggest cluster was headed by Attin $\mathrm{T}$ and included other 18 authors. However, the author with the highest number of links with other authors was Lussi A with a total of 36 links. Lussi A was the author with the highest number of papers and citations (14 papers; 1,642 citations). West NX and Addy $\mathrm{M}$ presented 11 papers in the top 100 list, retrieving 1,605 and 1,028 citations, respectively. Studies with 2-3 (40\%) and 4-6 (55\%) authors were more common than those with only one or more than six authors. The online supplementary Table 4 presents bibliometric indicators of the first ten authors with the highest number of papers in the list of the top 100 most cited.

Finally, 448 different keywords were retrieved from the 100 most-cited papers. The most frequent was "dental erosion" with 64 occurrences, followed by "erosion" (35), "enamel" (27), and "in vitro" and "enamel erosion" with 21 each. A keyword network map was created in VOSviewer to present the most frequent keywords throughout the decades (Fig. 2). It can be noticed that the keywords directly related to ETW, such as "dental erosion," "erosion," "enamel erosion," and "tooth wear" were all often observed after the 2000s (green color).

\section{Discussion}

This is the first bibliometric study to identify and discuss the top 100 most-cited papers related to ETW based on qualitative and quantitative approaches, demonstrating the field's scientific progress and highlighting its importance in dentistry, considering the high number of citations. In addition, mapping the knowledge generated in the field can be useful to understand the research activity and those papers that influence both research and practice [Mattos et al., 2021].

The most-cited paper [Jarvinen et al., 1991] received over 300 citations. Even though it was published in the 1990s, this paper presented the sixth-highest citation density. The impressive metrics of this study demonstrates its importance to this theme over the years. This 
paper may have been the most cited because it is one of the first case-control studies that analyzed nonindustrial risk factors associated with ETW, with relatively important sample size, considering it was conducted 30 years ago. As stated by the authors, more participants were investigated than in any previous study at that time. Knowing the risk factors of ETW is fundamental to prevent, diagnose, and treat this condition. Thus, it is very common that authors describe and discuss the etiology and the risk factors of ETW in papers, which may also explain the high citation rate.

In less broad research areas, papers with more than 100 citations can be considered highly cited papers, significantly influencing research and practice [Van Noorden et al., 2014; Santos et al., 2021]. In the present study, 39 papers were cited at least 100 times, confirming the relevance of ETW in dentistry. In this sense, a previous altimetric analysis [Adobes Martin et al., 2020] showed that ETW was one of the most searched themes in pediatric dentistry, probably given the frequent occurrence of these lesions also in younger patients [Jaeggi, and Lussi, 2014; Salas et al., 2015].

The 100 most-cited papers were published in a 66-year time course. The oldest paper was published in 1949 [Zipkin, and Mcclure, 1949], but most papers were published in the 2000s demonstrating the increased interest in this research topic in the last 20 years. It has been discussed that older papers tend to accumulate citations over time [Feijoo et al., 2014; Ahmad et al., 2019], but with advances in scientific knowledge, the introduction of new concepts and analysis may be related to the increased interest in more recent studies [Baldiotti et al., 2021; Mattos et al., 2021]. Furthermore, the analysis of citation density is an important indicator of the relevance of a paper. For instance, the 3rd most-cited paper in the present study, despite having 195 citations, compared to 330 citations of the 1st most cited, has a citation density of 21.67; that is, the paper was cited 21.67 times per year since its publication, compared to 11.38 from the 1st most cited.

Caries Research and The Journal of Dentistry were identified as the scientific journals with the highest number of papers in the top 100 most-cited list. Caries Research, the official journal of the European Organization for Caries Research, was founded in 1967 to promote research through epidemiological, clinical, and laboratorial studies on dental caries, fluorosis, erosion, and related dental diseases. The Journal of Dentistry is one of the leading international journals in the field of restorative dentistry. One of the main objectives is to influence dental practice. Both journals comprise highly relevant studies in ETW, presenting high numbers of citations, thus being understood and used as references to assist clinicians in making clinical decisions and for researchers in the interpretation of data and conducting research.

With a great emphasis on evidence-based practice, high-graded studies, namely, systematic reviews and randomized clinical trials, are expected to be valued [Patil et al., 2020]. Systematic reviews and randomized clinical trials may present a strong influence on researchers and practitioners, creating changes in clinical practice, representing the most reliable evidence [Murad et al., 2016]. However, only two systematic reviews (178 combined citations) and three clinical trials (237 combined citations) were compiled in the top 100 most-cited list, being placed in the second half of the list (out of the top 50 most cited). Laboratorial and observational studies were the most frequent observed study designs, followed by narrative literature reviews. These findings are in accordance with previous bibliometric analyses that analyzed the mostcited papers in dentistry [Feijoo et al., 2014], periodontology [Faggion et al., 2017], and cariology [Baldiotti et al., 2021]. The low number of systematic reviews and clinical trials identified in the top 100 list is an important gap that needs to be overcome. Most of the evidence on the topic is based on laboratorial and epidemiological studies.

Epidemiology (prevalence and risk factors) was the most addressed topic by the 100 most-cited papers in ETW, followed by the erosive potential of substances and anti-erosive/remineralizing agents. All studies evaluating remineralizing agents, for example, were laboratorial (in vitro) or controlled clinical simulations (in situ) studies. This information reinforces the aforementioned limitation about the lack of clinical trials. While it is understandable that it may be difficult to recruit a large sample of patients with ETW, data from small trials may be important to elucidate clinical questions and provide patients with the best treatment options.

Europe was the continent with the most number of papers in the list of the 100 most cited, as frequently reported in other bibliometric studies [Alarcón et al., 2017; Perazzo et al., 2019; Baldiotti et al., 2021]. This finding may be explained by the quality of European institutions and research centers, and the availability of funding for research development [Baldiotti et al., 2021]. The University of Bern in Switzerland was the leading institution in the top 100 most-cited papers. Besides the recognized quality of this university, this result is also related to the most productive author, Lussi A. The lack of representation of papers from African and Asian countries in the top 100 is also an important limitation in the field. Overcom- 
ing this issue is not simple and involves broad perspectives, such as public policies including governmental research funding and incentive. However, it could also express a lack of collaboration among authors from different continents. Perhaps, influent research centers could establish academic partnerships with less influent ones, contributing to the establishment and consolidation of new academic centers.

The six most prolific authors (with eight or more papers in the top 100 list) worked mainly on three topics related to ETW: epidemiology, the erosive potential of substances, and anti-erosive agents, thus emphasizing the search for the cause of this oral condition and the possibilities for intervention. Lussi A has developed over decades, together with his colleagues, essential studies on several aspects related to ETW, such as prevalence studies, diagnostic methods, chemical and histopathological characteristics, risk factors, and treatment of this condition. West NX, Addy M, and Hughes JA worked together on most of their authorship papers identified in the top 100 list. In the late 90s and early 2000s, they conducted a series of laboratorial studies, evaluating the erosive potential of dietary acids (juices and citrus fruits) and then carried out analyses of fluoride's role against ETW. Attin $\mathrm{T}$, in the 2000s, also carried out essential laboratorial studies mainly in the field of mineral anti-erosive agents such as sodium fluoride. Ganss C., from 2001 onward, carried out laboratorial studies focusing on anti-erosion mechanisms through the use of fluorides compounds in mouthwashes and toothpaste. Evaluating the VOSviewer coauthorship map, Lussi A, West NX, and Attin T headed the three biggest clusters of authors, demonstrating their importance and influence in the field of ETW.

Keywords are vital to identify papers through bibliographic search [Ahmad et al., 2019]. Selecting appropriate keywords is fundamental to retrieve relevant references, considering that most topics in dentistry present a vast number of papers. The most frequent keywords in the present analysis were "dental erosion," followed by "erosion." Another recurrent keyword was "enamel erosion," demonstrating that the research's main topic was evidenced by the authors. The term "erosive tooth wear," which has been introduced in the literature recently, did not appear as a keyword in the top 100 most-cited papers. It is understandable that more recent papers, that may have used "erosive tooth wear" as a keyword, may not have enough publication time to receive high citation rates. Surprisingly, "acid erosion" (with one occurrence) was the only outdated and discouraged term [Schlueter et al., 2020] related to ETW observed in the top 100 most- cited papers, in one paper from 2000 [Eisenburger et al., 2000]. Other discouraged terms such as "corrosive wear" or "bio-corrosion" did not appear in the keywords list.

Although citation rates are formally recognized as indicators of the relevance and merit of scientific papers [Hutchins et al., 2016; Perazzo et al., 2019], questions are often raised about their validity due to episodes of selfcitation [Moed, 2009]. However, although misconducts have been frequently observed, self-citation rates in the dental journals presented in the Journal Citation Reports were not correlated to the journals' impact factor [Livas, and Delli, 2018]. Furthermore, it is expected that authors who continually study specific topics presumably cite their own papers [Perazzo et al., 2019; Baldiotti et al., 2021].

In conclusion, the 100 most-cited papers related to ETW were published mainly by European countries, with laboratorial and observational study designs, addressing topics related to epidemiology, the erosive potential of substances, and anti-erosive agents. There is a gap concerning studies with higher levels of evidence. Therefore, researchers should focus their attention on conducting clinical trials, to provide the literature with more robust studies, with a higher level of evidence.

\section{Statement of Ethics}

Ethical approval was not required because the study was not conducted directly with humans, but analyzed data from the previous studies.

\section{Conflict of Interest Statement}

The authors have no conflicts of interest to declare.

\section{Funding Sources}

This study was supported in part by the Coordenação de Aperfeiçoamento de Pessoal de Nível Superior - Brasil (CAPES) - Finance Code 001.

\section{Author Contributions}

A.O. Rocha participated in data acquisition and interpretation, created the tables, and drafted and critically revised the manuscript. P.S. Santos participated in data acquisition and interpretation, created VOSviewer maps, and drafted and critically revised the manuscript. B.A. Machado participated in data acquisition and interpretation, and drafted and critically revised the manuscript. 
M. Bolan and M. Cardoso contributed to conception, design, data interpretation, and drafted and critically revised the manuscript. P.A. Martins-Júnior and C.M. Santana participated in data acquisition and interpretation, contributed to concept and design, and drafted and critically revised the manuscript. All authors gave their final approval and agreed to be accountable for all aspects of the work.

\section{Data Availability Statement}

All data generated or analyzed during this study are included in this article and its online supplementary material files. Further inquiries can be directed to the corresponding author.

\section{References}

Adobes Martin M, Zhou Wu A, Marques Martínez L, Gonzalvez Moreno AM, Aiuto R, Garcovich D. What is trending in paediatric dentistry? An Altmetric study on paediatric dentistry journals. Eur Arch Paediatr Dent. 2020;22(2):291-9.

Ahmad P, Dummer PMH, Chaudhry A, Rashid U, Saif S, Asif JA. A bibliometric study of the top 100 most-cited randomized controlled trials, systematic reviews and meta-analyses published in endodontic journals. Int Endod J. 2019;52:1297-316.

Ahmad P, Vincent Abbott P, Khursheed Alam M, Ahmed Asif J. A bibliometric analysis of the top 50 most cited articles published in the dental traumatology. Dent Traumatol. 2020; 36:89-99.

Alarcón MA, Esparza D, Montoya C, Monje A, Faggion CM. The 300 most-cited articles in implant dentistry. Int J Oral Maxillofac Implants. 2017;32:e1-8.

Baldiotti ALP, Amaral-Freitas G, Barcelos JF, Freire-Maia J, Perazzo MF, Freire-Maia FB, et al. The top 100 most-cited papers in cariology: a bibliometric analysis. Caries Res. 2021; 55(1):32-40.

Carvalho TS, Colon P, Ganss C, Huysmans MC, Lussi A, Schlueter N, et al. Consensus report of the European Federation of Conservative Dentistry: erosive tooth wear - diagnosis and management. Clin Oral Investig. 2015;19(7): 1557-61.

Eisenburger M, Hughes J, West NX, Jandt KD, Addy M. Ultrasonication as a method to study enamel demineralisation during acid erosion. Caries Res. 2000;34(4):289-94.

Faggion CM, Málaga L, Monje A, Trescher AL, Listl S, Alarcón MA. The 300 most cited arti- cles published in periodontology. Clin Oral Investig. 2017;21:2021-8.

Feijoo JF, Limeres J, Fernández-Varela M, Ramos I, Diz P. The 100 most cited articles in dentistry. Clin Oral Investig. 2014;18:699-706.

Hutchins BI, Yuan X, Anderson JM, Santangelo GM. Relative citation ratio (RCR): a new metric that uses citation rates to measure influence at the article level. PLoS Biol. 2016;14: e1002541.

Jaeggi T, Lussi A. Prevalence, incidence and distribution of erosion. Monogr Oral Sci. 2014; 25:55-73.

Jarvinen VK, Rytomaa II, Heinonen OP. Risk factors in dental erosion. J Dent Res. 1991;70:942-7.

Jayaratne YS, Zwahlen RA. The evolution of dental journals from 2003 to 2012: a bibliometric analysis. PLoS One. 2015;10:e0119503.

Kear R, Colbert-Lewis D. Citation searching and bibliometric measures: resources for ranking and tracking. Crln. 2011;72(8):470-4.

Livas C, Delli K. Journal self-citation rates and impact factors in dentistry, oral surgery, and medicine: a 3-year bibliometric analysis. J Evid Based Dent Pract. 2018;18:269-74.

Mattos FF, Perazzo MF, Vargas-Ferreira F, Martins-Júnior PA, Paiva SM. Top 100 most-cited papers in core dental public health journals: bibliometric analysis. Community Dent Oral Epidemiol. 2021;49:40-6.

Moed HF. New developments in the use of citation analysis in research evaluation. Arch Immunol Ther Exp (Warsz). 2009;57:13-8.

Murad MH, Asi N, Alsawas M, Alahdab F. New evidence pyramid. Evid Based Med. 2016;21: 125-7.

Patil SS, Sarode SC, Sarode GS, Gadbail AR, Gondivkar S, Kontham UR, et al. A biblio- metric analysis of the 100 most cited articles on early childhood caries. Int J Paediatr Dent. 2020;30:527-35.

Perazzo MF, Otoni ALC, Costa MS, GranvilleGranville AF, Paiva SM, Martins-Júnior PA. The top 100 most-cited papers in paediatric dentistry journals: a bibliometric analysis. Int J Paediatr Dent. 2019;29:692-711.

Salas MMS, Nascimento GG, Huysmans MC, Demarco FF. Estimated prevalence of erosive tooth wear in permanent teeth of children and adolescents. An epidemiological systematic review and meta-regression analysis. J Dent. 2015;43:42-50

Santos PS, Santos N, Moccelini BS, Bolan M, Santana CM, Martins-Junior PA, et al. The top 100 most-cited papers authored by Dr. Jens Ove Andreasen: a bibliometric analysis. Dent Traumatol. 2021;37(3):365-82.

Schlueter N, Jaeggi T, Lussi A. Is dental erosion really a problem? Adv Dent Res. 2012;24:6871.

Schlueter N, Amaechi BT, Bartlett D, Buzalaf MAR, Carvalho TS, Ganss C, et al. Terminology of erosive tooth wear: consensus report of a workshop organized by the ORCA and the cariology research group of the IADR. Caries Res. 2020;54:2-6.

Van Eck NJ, Waltman L. Citation-based clustering of publications using CitNetExplorer and VOSviewer. Scientometrics. 2017;111:105370.

Van Noorden R, Maher B, Nuzzo R. The top 100 papers. Nature. 2014;514:550-3.

Zipkin I, Mcclure FJ. Salivary citrate and dental erosion. Procedure for determining citric acid in saliva-dental erosion and citric acid in saliva. J Dent Res. 1949;28:613-26. 